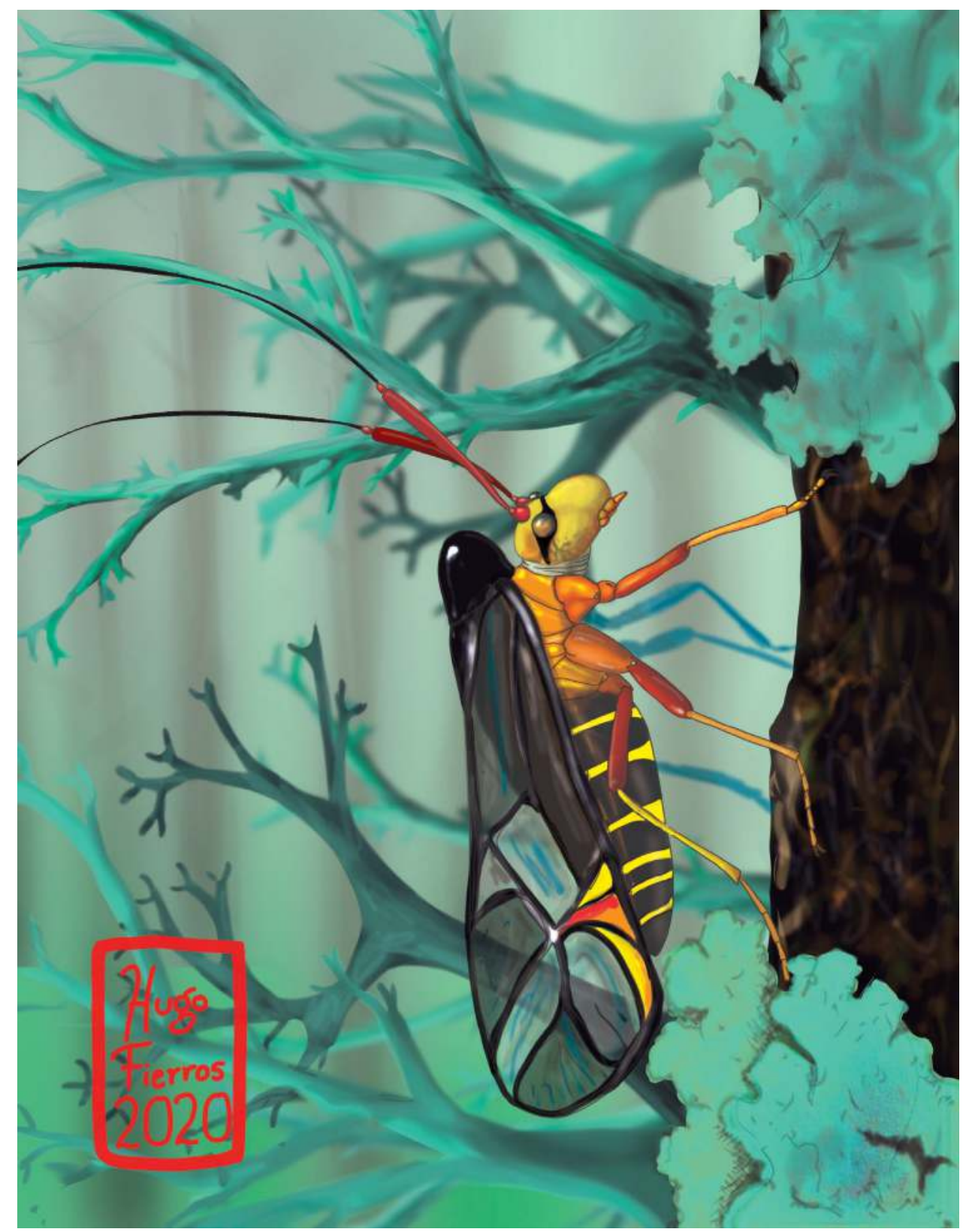

Dugesiana, Año 27, No. 2, julio 2020-diciembre 2020 segundo semestre de 2020), es una publicación semestral, editada por la Universidad de Guadalajara, a través del Centro de Estudios en Zoología, por el Centro Universitario de Ciencias Biológicas y Agropecuarias. Camino Ramón Padilla Sánchez \# 2100, Nextipac, Zapopan, Jalisco, Tel. 37771150 ext. 33218, http://148.202.248.171/dugesiana/index.php/DUG/index, glenusmx@gmail.com. Editor responsable: José Luis Navarrete-Heredia. Reserva de Derechos al Uso Exclusivo 04-2009-062310115100203, ISSN: 2007-9133, otorgados por el Instituto Nacional del Derecho de Autor. Responsable de la última actualización de este número: José Luis Navarrete-Heredia, Editor y Ana Laura González-Hernández, Asistente Editorial. Fecha de la última modificación 1 de julio 2020, con un tiraje de un ejemplar.

Las opiniones expresadas por los autores no necesariamente reflejan la postura del editor de la publicación.

Queda estrictamente prohibida la reproducción total o parcial de los contenidos e imágenes de la publicación sin previa autorización de la Universidad de Guadalajara. 


\title{
Nueva especie de ácaro del género Phyllozetes Gordeeva, 1978 (Acari: Oribatida: Cosmochtho- niidae) de Coahuila, México
}

\author{
A new species of the mite genus Phyllozetes Gordeeva, 1978 (Acari: Oribatida: Cosmochthoniidae) \\ from Coahuila, México
}

\author{
Margarita Ojeda, Leonardo García, Jair Páez y José G. Palacios-Vargas* \\ Laboratorio de Sistemática y Ecología de Microartrópodos, Departamento de Ecología y Recursos Naturales, \\ Facultad de Ciencias, UNAM, 04530. *Autor para correspondencia: José G. Palacios-Vargas. E-mail: troglo- \\ laphysa@hotmail.com
}

\section{RESUMEN}

Se describe Phyllozetes aldretei sp. nov. un ácaro cosmoctónido de suelos oligotróficos en el Desierto Chihuahuense de México. Esta nueva especie se diferencia del resto de sus congéneres por poseer las sedas rostrales birrámeas, con sus ramas bifidas, en forma de "V" y conectadas por una membrana hialina. Adicionalmente, la combinación de sedas eréctiles de las líneas $e$ y $f$ con numerosas y largas barbulaciones marginales, distancia entre las sedas $d 1-d 2$ menos del doble que la $d 1-d 1$, y la fórmula coxiesternal 3-3-3-4 son caracteres diagnósticos de la nueva especie.

Palabras clave: Oribatei, Enarthronota, Cuatro Ciénegas.

\begin{abstract}
Phyllozetes aldretei sp. nov. Cosmochthoniid mite, from oligotrophic soils in the Chihuahuan Desert of México is described. This new species differs from the rest of its congeners by possessing birramea rostral setae, with their bifid branches "V" shape and connected by a hyaline membrane. Additionally, the combination of the erectile setae $e$ and $f$ lines with numerous and long marginal barbulations, distance between the $d 1-d 2$ setae less than twice that of $d 1-d 1$, and the 3-3-3-4 coxiesternal formula are diagnostic characters of the new species.
\end{abstract}

Keywords: Oribatei, Enarthronota, Cuatro Ciénegas.

Los ácaros oribátidos Enarthronota son una de las supercohortes morfológicamente más diversas (Norton, 2001). Presentan un notogáster con 1-3 suturas transversales: una sutura de tipo E (articulación simple entre placas) entre las sedas de las líneas $c y d$; y 2 suturas tipo $\mathrm{S}$ (espacio entre las placas con 4 escleritos intercalares pequeños, cada uno con una seda). Sedas eréctiles largas en las líneas $e y f$; y sutura tipo L (especialización defensiva, en donde dos placas pueden moverse para proteger la amplia zona de articulación) (Norton \& Behan-Pelletier, 2009).

Dentro de este grupo, la familia Cosmochthoniidae incluye ácaros de talla pequeña a mediana $(100 \mu \mathrm{m})$, de color pálido a café claro, que poseen un cuerpo dividido por 3 suturas transversales, de tipo E y S. Michael (1885) los describió traducido como sigue "los segmentos son capaces de cierta extensión y retracción telescópica, y con esto, las sedas dorsales del cuerpo pueden adoptar una posición eréctil, como lo hacen los puerco espines". Su posición dentro de Oribatida es incierta, debido al número de plesiomorfías, la presencia de sedas eréctiles, su aparente reproducción asexual y la falta de caracteres derivados (Norton \& Behan-Pelletier, 2009).

A la fecha Cosmochthoniidae es una familia conformada por 47 especies (Subías, 2020), de siete géneros:
Cosmochthonius Berlese, 1910 (31 spp.); Gozmania Balogh \& Mahunka, 1983 (3 spp.); Krivolutskiella Gordeeva, 1980 (2 spp.); Nipponiella Gordeeva, 1980 (1 spp.); Trichthonius Hammer, 1961 (1 spp.) y Phyllozetes Gordeeva, 1978 (10 spp.).

Phyllozetes con 10 especies, es el segundo género más diverso de la familia. Phyllozetes emmae (Berlese, 1910) es la especie tipo; Phyllozetes alatus Mahunka, 1982 se conoce de Etiopia y el Congo; Phyllozetes hypoquercus McDaniel \& Bolen, 1980 de Texas, Estados Unidos de Norteamérica; Phyllozetes latifolius Gordeeva, 1980 de Indonesia y el Mediterráneo; Phyllozetes longifolius Balakrishnan, 1986 y Phyllozetes nilambricus Balakrishnan, 1986 de la India (Balakrishnan, 1986); Phyllozetes heelei Shereef, Hanna \& Duweini, 1981 de Egipto; Phyllozetes osithchnjukovi Gordeeva, 1980 del Mediterráneo oriental; Phyllozetes tauricus Gordeeva, 1978 del Mediterráneo y Phyllozetes subiasi Jorrin, 2014 de España.

Para México, Vazquez et al. (2016) citan al género con la especie P. latifolius en suelos de Quintana Roo.

Phyllozetes puede encontrarse habitando en las capas superiores de suelos debajo de vegetación xerófita, mostrando adaptaciones a estos ambientes, como el tamaño pequeño y las sedas del notogáster largas y foliadas, lo 
que les ayuda a reducir la pérdida de agua a través de la superficie del cuerpo (Gordeeva, 1980). Norton (2001) y Seniczak et al. (2011) han señalado que la capacidad de erección y modificación de las sedas dorsonotales es una adaptación de protección en contra de sus depredadores.

La descripción de esta nueva especie es parte del inventario de los microartrópodos del suelo del Churince, Cuatro Ciénegas, Coahuila, México; estudio que inició en 2015 para conocer la diversidad de los ácaros edáficos asociados a 5 tipos de vegetación presentes en el valle.

\section{MATERIALES Y MÉTODOS}

Sitio de estudio. El Valle de Cuatro Ciénegas (VCC) es un área pequeña (aproximadamente de $1,500 \mathrm{~km}^{2}$ ) ubicada en la parte central del estado de Coahuila. A pesar de ser una de las zonas más secas del Desierto Chihuahuense (con menos de $200 \mathrm{~mm}$ de lluvia anual), se estima que alberga alrededor de 200 cuerpos de agua (pozas, lagunas, playas, ciénegas, ríos naturales y canales hechos por el hombre (Dinger et al. 2005), que albergan una variedad de especies endémicas (Souza et al. 2006, 2012; Desnues et al. 2008; Álvarez \& Ojeda 2019). El clima en el VCC es generalmente cálido y árido, con temperaturas extremas que alcanzan los $45^{\circ} \mathrm{C}$ en julio, y bajo $0^{\circ} \mathrm{C}$ en el mes de enero (Conagua 2015). Particularmente, el sistema del Churince $\left(26^{\circ} 50^{\prime} \mathrm{N} ; 10^{\circ} 28^{\prime} \mathrm{W}\right)$ comprende un manantial perenne, una poza (Poza Churince), una laguna (Laguna Intermedia o Los Güeros) y una playa (Laguna Grande) que están conectados por corrientes cortas y poco profundas (López-Lozano et al. 2012). En el valle se pueden observar 5 tipos de vegetación predominantes: Peladero sin cubierta vegetal dominante, solo algunos ejemplares de Sporobolus rodeados de dunas de arena; Sotol en donde Dasyrilion wheeleri S.Wat ex Rothr., está bien distribuído; Tular con Distichlis spicata (L.) Greene; Larrea (gobernadora) con Larrea tridentata (Sessé \& Moc. ex DC.) Coville, dominando el área, y el Mezquital con Prosopis glandulosa Torr. En general los suelos presentan una alta alcalinidad y bajas concentraciones de fósforo, lo que hace este sitio una zona oligotrófica.

Muestreo. Los ejemplares de Phyllozetes aldretei sp. nov. utilizados en este trabajo se obtuvieron de muestras de suelo de 3 de los 5 distintos tipos de vegetación definidos en el área (detalles ver Ojeda \& Gasca, 2019) en 4 visitas realizadas durante 2015-2016 al Churince, Valle de Cuatro Ciénegas, Coahuila, México. La separación de los ácaros de las muestras se realizó mediante el método del embudo de Berlese-Tulgreen y posterior flotación en solución agua-jabón líquido $(\mathrm{D}=250 \mathrm{gr} / 500 \mathrm{ml})$. Los ácaros se aclararon en ácido láctico y se montaron en preparaciones microscópicas semipermanentes en líquido de Höyer. Las medidas se dan en micrómetros $(\mu \mathrm{m})$, y las ilustraciones se realizaron con ayuda de un microscopio de contraste de fases Zeiss, con un tubo de dibujo adaptado. La longitud del cuerpo fue medida en vista lateral, desde la punta del rostro hasta el borde posterior de la placa ventral. El ancho del notogáster, se refiere al ancho máximo en vista dorsal a nivel de segmento donde se insertan las sedas $f$. La quetotaxia de las patas se da de acuerdo con la secuencia trocánter-fémur-genua-tibia-tarso (fámulus incluído), la fórmula de los solenidios de las patas entre paréntesis de acuerdo con la secuencia genua-tibia-tarso. La terminología general sigue la de Norton \& Behan-Pelletier (2009). Los ejemplares fueron colectados con el permiso (FAUT-0230) emitido a V. Souza (FAUT-0230) por SEMARNAT. El material se deposita en la Colección Nacional de Ácaros (CNAC) del Instituto de Biología, UNAM, México y la Colección de oribátidos del Laboratorio de Ecología y Sistemática de Microartrópodos (LESM), Facultad de Ciencias, UNAM, México.

\section{RESULTADOS}

Phyllozetes Gordeeva, 1978 Diagnosis

Especie tipo: Cosmochthonius emmae Berlese, 1910

Rostro fenestrado; notogáster dividido en 4 segmentos por la presencia de tres suturas en el histerosoma; sedas de la serie- $d$ asociadas a la sutura-E; sedas $e$ y $f$ foliáceas y eréctiles; patas polidáctilas, heterodáctilas; fórmula tarsal 2-2-2-3 (Gordeeva, 1978).

\section{Phyllozetes aldretei $\mathbf{s p . ~ n o v . ~}$}

(Figuras 1-11)

http://zoobank.org/33DE0AA2-5818-4E57-A461527BC3833CCD

Descripción del adulto. Holotipo: Largo 247.5, ancho 117.5. Paratipos $(n=10)$ largo 232.7 (200 - 247.5); ancho 106.4 (100 - 117.5), altura 76.7 (64.4 - 85). Ácaros de talla pequeña, longitud del idiosoma ( $232 \mu \mathrm{m}$ promedio) 2.2 veces el ancho máximo, poco esclerosados, con cutícula transparente y cerotegumento con microfoveólas en las placas dorsales y laterales.

Prodorso (Fig. 1). Tectum con perforaciones rostrales (fenestrado), arregladas en 5-7 hileras longitudinales, cada una conformada por 2-4 fenestras, la más posterior ovalada. Sedas rostrales (ro) birrámeas con sus ramas bifidas, en forma de "V" y conectadas por una delgada membrana un tanto hialina cuya observación es más evidente en el extremo distal que une las ramas y termina formando una "V" (Fig. 3A), ramas profusamente pectinadas tanto dorsal como lateralmente, con el extremo en punta. Sedas lamelares $(l a)$ birrámeas, en forma de " $\mathrm{T}$ ", brazo anterior más largo que el posterior (Fig. 3B); sedas exobotridiales (exa) gruesas, pectinadas hacia la cara exterior (Fig. 3C); seda exp más corta, ligeramente barbulada (Fig. 3D). Sedas interlamelares $(\mathrm{in})$ pectinadas en la cara dorsal exterior, situadas posteriormente del botridio (Fig. 3E). Sensila botridial masuda, barbulada, con el pedicelo aserrado sólo después del primer tercio (Fig. 3F).

Pedipalpos: con cinco artejos, fémur con dos sedas, genua con 1, tibia con 3. Quetotaxia del tarso con 9 y un solenidio (Fig. 4).

Quelíceros: Dedo fijo más corto y ancho que el dedo 
móvil, terminando en 4 dientes ( 2 pequeños y 2 más largos), seda chb pectinada, ancha en su porción distal, seda cha lisa y larga (Fig. 5). Dedo móvil delgado y terminando en un diente que sobresale el nivel del dedo fijo.

Notogáster (Figs. 1, 6 y 7). Cuerpo dividido en cuatro regiones dorsales; placa anterior NA (27.4), con doble de ancho que las placas posteriores NM1 y NM2 (14.7 y 13.4 respectivamente). sedas de la placa NA barbuladas, $c 1$ ligeramente más pequeña que el resto de las sedas (22.1); sedas $c 2, c 3$ y $c p(26.9,26.6$ y 25.8 respectivamente). sedas $c 1, c 2$ y $c 3$ alineadas a la misma altura de la placa NA, seda $c p$ desplazada hacia la zona lateral, cerca de la hilera $d$. NM1 con sedas barbuladas, $d 1$ más corta que $d 2$, (14.6 y 24.4 respectivamente); ambas sedas unidas por un pliegue en el primer surco notogastral, con una separación $d 1-d 1$ de 7.2 , menor que la distancia $d 1-d 2$ (11.9). sedas de las series $e$ y $f$ subiguales (98-104), foliáceas con los primeros $2 / 3$ más ensanchados que el tercio final, cada una con un eje central bien definido; margen de la seda con una serie de largas barbulaciones (6-9 $\mu \mathrm{m})$, separación entre éstas de 2 $\mu \mathrm{m}$, (Fig. 3G). Sedas $h$ y $p s$ ubicadas en el margen posterior de la placa PYG, de tamaño medio y pectinadas hacia la cara exterior, las posteriores más pectinadas.

Vista lateral del Notogáster (Figs. 6 y 7). Por debajo de la placa NA se observa una pequeña placa triangular, y una pequeña estructura cóncava triangular humeral $(h f l)$ (solapa humeral). Por debajo de $h f l$ y sobre el acetábulo III hay 2 placas accesorias pequeñas (apll y apl2, esta última se sobrelapa con apll (no visible en el dibujo). Por encima del acetábulo IV apl3 de forma alargada. La placa PLm se encuentra por debajo de las placas medias (Nmly $N m 2)$ y la pigidial $(P Y G)$, y en ella se observa la lirifisura $i m$, así como pequeñas foveólas en su superficie.

Región Ventral (Fig. 2). Placas epimerales separadas, con sedas cortas y con pocas ramificaciones o pectenes. Los epímeros III y IV fusionados en su parte media basal. Fórmula coxiesternal I-IV: 3-3-3-4. Placa genital con 10 pares de sedas barbuladas y largas, distribuidas en dos líneas longitudinales irregulares; 6 pares ubicados en la parte media y 4 pares desplazados lateralmente, las últimas sedas ligeramente más largas. Placa anal con 4 pares de sedas pectinadas (holotipo con 5 sedas en uno de los lados). Placa adanal posteriormente terminando en forma de " $U$ " con cuatro pares de sedas adanales, sedas posteriores más pectinadas (Figs. 2 y 7), como lo descrito para Phyllozetes subiasi Jorrin (2014).

Patas (Figs. 8-11). Tarsos I-IV con la fórmula de uñas típica del género 2-2-2-3, uñas laterales más delgadas que la central y ligeramente curvadas hacia abajo. Quetotaxia de las patas (fémur a tarso, famulus incluído), solenidios entre paréntesis: Pata I: 5-5-5 (1)-17 (2), Pata II: 5-5-6 (1) -15(2), Pata III: 3- 4- 4 (1)-14; Pata IV: 3- 4- 4 (1)-12.

Material tipo. Holotipo (Pe0054) depositado en la colección LESM (con código LESM-1560), 8 paratipos (Pe0006, Pe0055, Pe0063, Pe0065, So0028, So0100, La0002, La0045) en la colección LESM (códigos LESM-
1561-1569), y dos (Pe0004, So 0032) en la CNAC (códigos CNAC011253 y 4). Serie tipo en preparaciones semipermanentes en Höyer.

MÉXICO: Coahuila, Cuatro Ciénegas, Churince. Pe0006, Pe0054, Pe0055, Pe0063, Pe0065, $26^{\circ} 51.018 \mathrm{~N} / 102^{\circ} 08.897 \mathrm{~W}$, 6-IX-2015, $760 \mathrm{msnm}$ suelo con vegetación predominante de Sporobolus sp., M. Ojeda col. So0028, S00032, $26^{\circ} 50.921 \mathrm{~N} / 102^{\circ} 08.591 \mathrm{~W}, 14-\mathrm{V}-$ 2016, So0100, 3-X-2016, 760 msnm suelo con vegetación predominante de Dasylirion wheeleri, M. Ojeda col. La0002, La0045, 26 $50.361 / 102^{\circ} 07.985,14-\mathrm{V}-2016,775 \mathrm{msnm}$ suelo con vegetación predominante de Larrea tridentata, M. Ojeda col. Pe0113, Pe0127, 26 $6^{\circ} 51.018 \mathrm{~N} / 102^{\circ} 08.897 \mathrm{~W}$, 3-X-2015, $760 \mathrm{msnm}$ suelo con vegetación predominante de Sporobolus sp, M. Ojeda y A. Carlos col. So0100, $26^{\circ} 50.921 \mathrm{~N} / 102^{\circ} 08.591 \mathrm{~W}, 3-\mathrm{X}-2016,760 \mathrm{msnm}$ suelo con vegetación predominante de Dasylirion wheeleri, M. Ojeda y A. Carlos col.

Etimología. Se dedica esta especie al Dr. Alfonso Neri García Aldrete en homenaje a su extenso trabajo en la Sistemática y taxonomía de los Psocoptera de México.

\section{DISCUSION}

Phyllozetes aldretei $\mathbf{s p . ~ n o v . ~ s e ~ d i f e r e n c i a ~ d e l ~ r e s t o ~ d e ~}$ sus congéneres por poseer las sedas rostrales birrámeas, con sus ramas bífidas en forma de "V", conectadas por una membrana y la fórmula coxiesternal 3-3-3-4 son los caracteres diagnósticos de esta nueva especie. Además de la combinación de las sedas eréctiles de las líneas $e$ y $f$ con numerosas y largas barbulaciones marginales, que terminan en su porción distal de manera muy estrecha; y distancia entre las sedas $d 1-d 2$ menos del doble que la $d 1-d 1$.

Con respecto a las dos especies presentes en el Continente Americano, Phyllozetes aldretei sp. nov. se diferencia de $P$. hypoquercus, ya que en esta última la lámina hialina entre las sedas rostrales está ausente; las sedas e yf no terminan en una fina punta y las barbulaciones del borde son más pequeñas. Además de que su longitud no alcanza el borde posterior del cuerpo, y la fórmula epimeral que es 3-3-3-3 (figuras 1 y 2 de McDaniel \& Bolen, 1980).

Por su parte con P. latifolius registrada por Vazquez et al. (2016), se distingue por que la talla es más pequeña (160 x 60), hay un número mayor de fenestras en el rostro, las sedas $e$ y $f$ tienen distinto grosor, siendo las sedas $f$ más asimétricas y el doble del ancho de las sedas $e$, además de que la longitud de estas sedas excede el borde posterior del cuerpo en casi un tercio de su longitud (figural de Goordeva, 1980).

De entre las especies descritas a la fecha, Phyllozetes aldretei sp. nov. es semejante a $P$. nilambricus, al poseer una talla similar (cerca de $230 \mu \mathrm{m}$ ), seda rostral apicalmente birrámea, seda lamelar en forma de " $T$ ", sedas $e \mathrm{y} f$ de forma foliácea y mismo número de sedas genitales. Las barbulaciones de las sedas $e$ y $f$ en $P$. aldretei sp. nov. miden entre 6 y $9 \mu \mathrm{m}$, y aunque en la descripción original de P. nilambricus no se hace mención de la longitud de sus 
barbulaciones, estas se observan con una menor longitud relativa (figuras 8-11 de Balakrishnan, 1986 ). La nueva especie puede ser distinguida de P. nilambricus y del resto de sus especies congenéricas ya que es la única especie con fórmula epimeral I-IV 3-3-3-4 (3-2-3-4 en el resto de las especies) y la seda rostral con una delgada membrana que une las dos ramas. Adicionalmente, $P$. aldretei sp. nov. se diferencia de $P$. nilambricus por la distancia entre las setas $d 1-d 2$ que es menor del doble de la distancia entre d1-d1 (más del doble en $P$. nilambricus); la longitud de las sedas $e$ y $f$ es menor (100 en $P$. aldretei 100-130 en $P$. nilambricus), y por la presencia de 5-7 hileras de fenestras en el rostro cada una con sólo 2-4 hendiduras, a diferencia de $P$. nilambricus que se menciona tiene 8-9 .

\section{AGRADECIMIENTOS}

El primer autor agradece a Valeria Souza y Luis Eguiarte por la invitación a participar en el inventario de la fauna de Cuatro Ciénegas. También agradecemos los invaluables comentarios y sugerencias de los dos revisores anónimos. Este trabajo estuvo apoyado por el proyecto Alianza WWFFundación Carlos Slim a VS y LE. Leonardo García (LG) contó con una beca como ayudante de Investigador del Dr. Palacios-Vargas en el SNI (CONACyT, México).

\section{LITERATURA CITADA}

Álvarez, F. \& M. Ojeda. 2019. Chapter I: The fauna of Cuatro Ciénegas Basin, A Unique Assemblage of Species, Habitats, and Evolutionary Histories. En: Álvarez, F. \& Ojeda, M. (Eds.) Animal Diversity and Biogeography of the Cuatro Ciénegas Basin. Springer International Publishing, Springer Nature Switzerland AG, pp.1-10.

Ojeda, M. \& J. Gasca-Pineda. 2019. Chapter 3: Abundance and Diversity of the Soil Microarthropod Fauna from the Cuatro Ciénegas Basin. En: Álvarez, F. \& Ojeda, M. (Eds.) Animal Diversity and Biogeography of the Cuatro Ciénegas Basin. Springer International Publishing, Springer Nature Switzerland AG pp. 29-51.

Balakrishnan, M.M. 1986. Two new species of the genus Phyllozetes (Acari: Orobatei: Cosmochthoniidae) from Kerala. Geobios new Reports 5: 15-18

Conagua, SMN. 2015. Normales climatológicas por estación. Ciudad de México: Servicio Meteorológico Nacional, CONAGUA. Available at http://smn.cna.gob. mx/es/ (accessed 11 March 2019).

Desnues, C., B. Rodriguez-Brito, S. Rayhawk, S. Kelley, T. Tran, M. Haynes, \& F. Rohwer. 2008. Biodiversity and biogeography of phages in modern stromatolites and thrombolites. Nature 452:340-343. doi: 10.1038/ nature 06735

Dinger, E.C., A. E. Cohen, D. A. Hendrickson, \& J. C. Marks. 2005. Aquatic Invertebrates of Cuatro Ciénegas, Coahuila, México: Natives and Exotics. The Southwestern Naturalist 50 (2): 237-281

Gordeeva E.V. 1978. A new genus of oribatid mites from eastern Crimea. Zoologicheskii Zhurnal. 57(7): 10991101

Gordeeva E.V. 1980. Oribatid mites of the family Cosmochthoniidae (Oribatei) Zoologicheskii Zhurnal. 59(6): 838-850 (en Ruso).

Jorrin, J. 2014. Two new arthronotic mites from the South of Spain (Oribatida, Cosmochthoniidae), with a new subgenus and species of Cosmochthonius and one new species of Phyllozetes. Acarologia 54 (2): 411-419.

López-Lozano, N.E., L. E. Eguiarte, G. Bonilla-Rosso, F. García-Oliva, C. Martínez-Piedragil, C. Rooks \& V. Souza. 2012. Bacterial communities and the nitrogen cycle in the gypsum soil of Cuatro Ciénegas Basin, Coahuila: a Mars analogue. Astrobiology 12:699-709. DOI: 10.1089/ast.2012.0840.

McDaniel, B. \& E. G. Bolen. 1980. A new species of Phyllozetes Gordeeva from Texas (Cryptostigmata: Cosmochthoniidae). International Journal of Acarology, 6(3): 225-228.

Michael A.-D. 1885. New British Oribatidae. Journal of the Royal Microbiological Society., 5: 385-397. DOI:10.1111/j.1365-2818.1885.tb05787.x

Norton, R. A. (2001). Systematic relationships of Nothrolohmanniidae, and the evolutionary plasticity of body form in Enarthronota (Acari: Oribatida) - In: Halliday R. B., Walter D. E., Proctor H. C., Norton R. A., Colloff M. J. (Eds.). Acarology: Proceedings of the 10th International Congress. Melbourne: CSIRO Publishing. p. 58-75.

Norton, R.A. \& V. M. Behan-Pelletier. 2009. Oribatida. Chapter 15. In: Krantz, G.W. \& Walter, D.E. (eds.). A Manual of Acarology. Texas Tech University Press, Lubbock. pp. 430-564.

Seniczak S., R. Penttinen \& A. Seniczak. 2011. The ontogeny of morphological traits in three European species of Cosmochthonius Berlese, 1910 (Acari: Oribatida: Cosmochthoniidae). Zootaxa, 3034: 1-31. DOI: 10.11646/zootaxa.3034.1.1

Souza, V., L. Espinosa-Asuar, A. E. Escalante, L. E. Eguiarte, J. Farmer, L. Forney, L. Lloret, J. M. RodríguezMartínez, X. Soberón, R. Dirzo \& J. J. Elser. 2006. An endangered oasis of aquatic microbial biodiversity in the Chihuahuan desert. Proceedings of the National Academy of Sciences of the United States of America 103 (17) 6565-6570. DOI: 10.1073/pnas.0601434103

Souza, V., J. Siefert, A.E. Escalante, J. J. Elser \& L. E. Eguiarte. 2012. The Cuatro Cienegas Bolson in Coahuila, Mexico: an astrobiological Precambrian park. Astrobiology 12:641-647. DOI: 10.1089/ast.2011.0675

Subías, L.S. 2020. Listado sistemático, sinonímico y biogeográfico de los ácaros oribátidos (Acariformes: Oribatida) del mundo (excepto fósiles). (15 actualización). 527 pp. Disponible en: http://bba. bioucm.es/cont/docs/RO_1.pdf

Vázquez, G. M.M., D. A. May Uicab \& E. B. Alamilla Pastrana. 2016. Riqueza específica y biodiversidad 
de Cozumel Quintana Roo, México. Teoría y Praxis, Recibido: 5 mayo 2020

19: 137-171 [fecha de Consulta 13 abril de 2020]. Aceptado: 16 junio 2020

Disponible en https://www.redalyc.org/articulo. oa? id $=456146535008$.

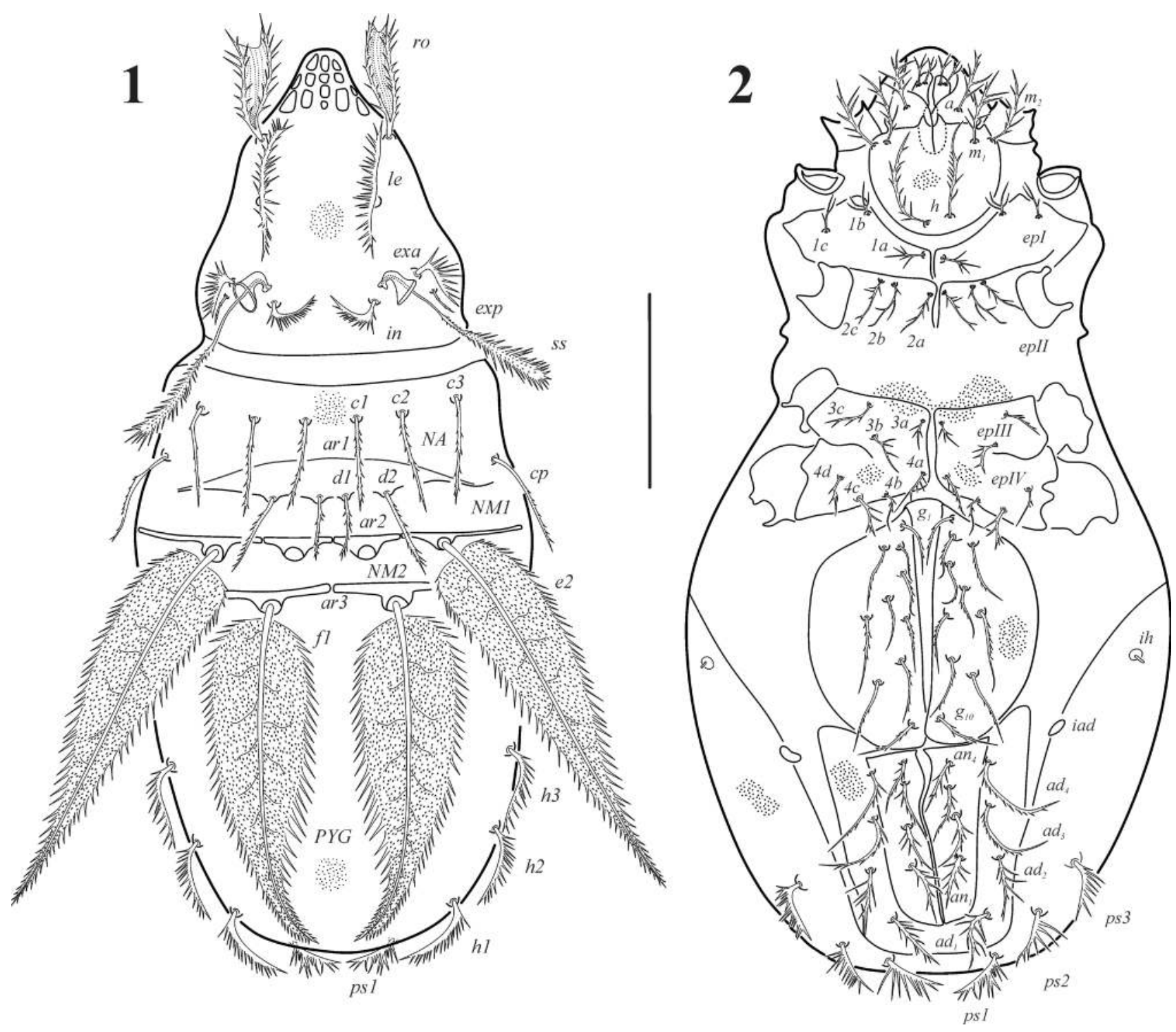

Figuras 1-2. Phyllozetes aldretei sp. nov. 1, vista dorsal del cuerpo; 2, vista ventral del cuerpo. Escala $50 \mu \mathrm{m}$. 


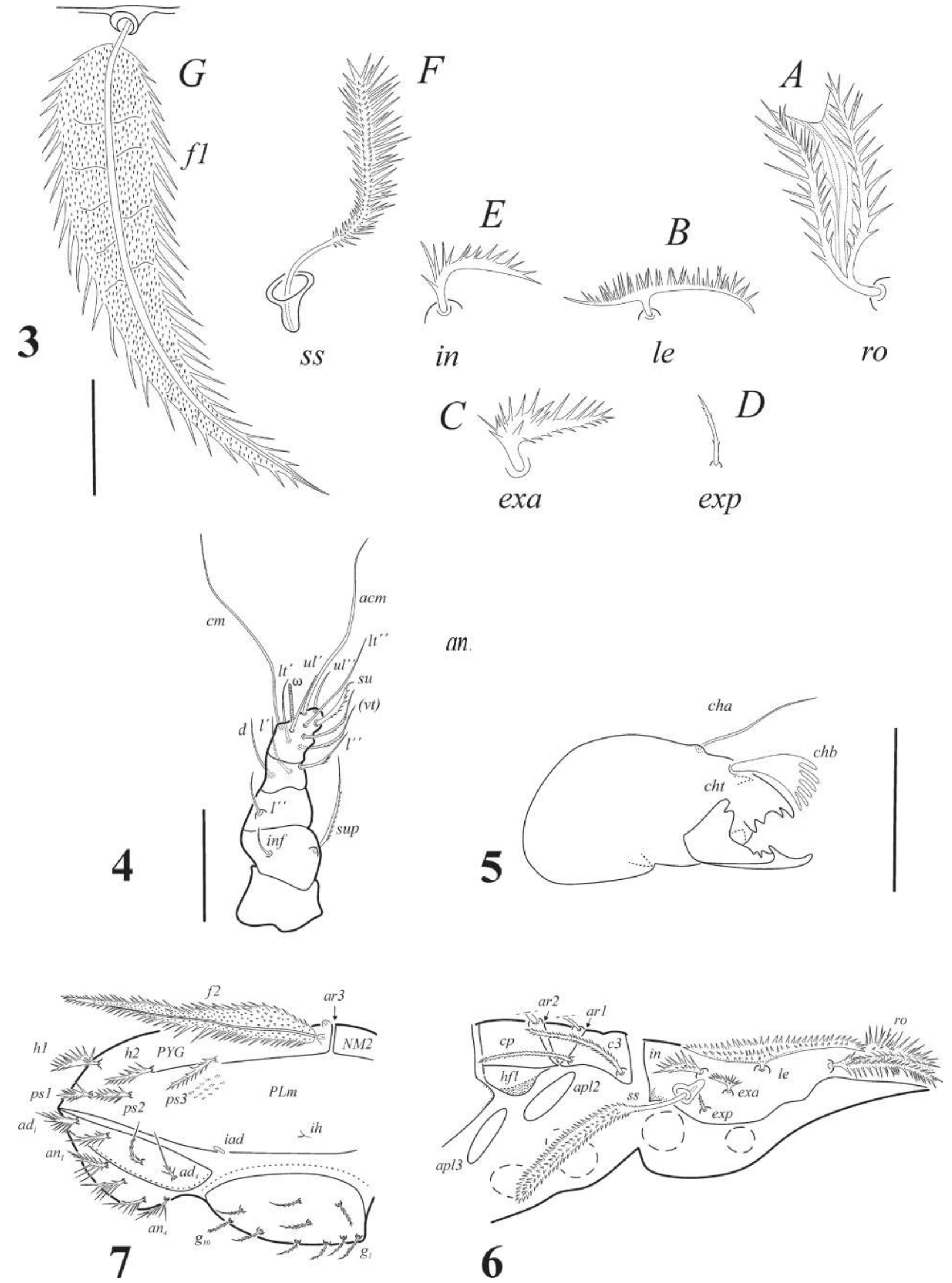

Figuras 3-7. Phyllozetes aldretei sp. nov. 3A-F, sedas del prodorso, 3G seda $f$, vista dorsal; 4, pedipalpo; 5, quelícero; 6 , vista lateral del prodorso y parte anterior del notogáster; 7, vista lateral de la parte anterior del notogáster. Escala $20 \mu \mathrm{m}$. 


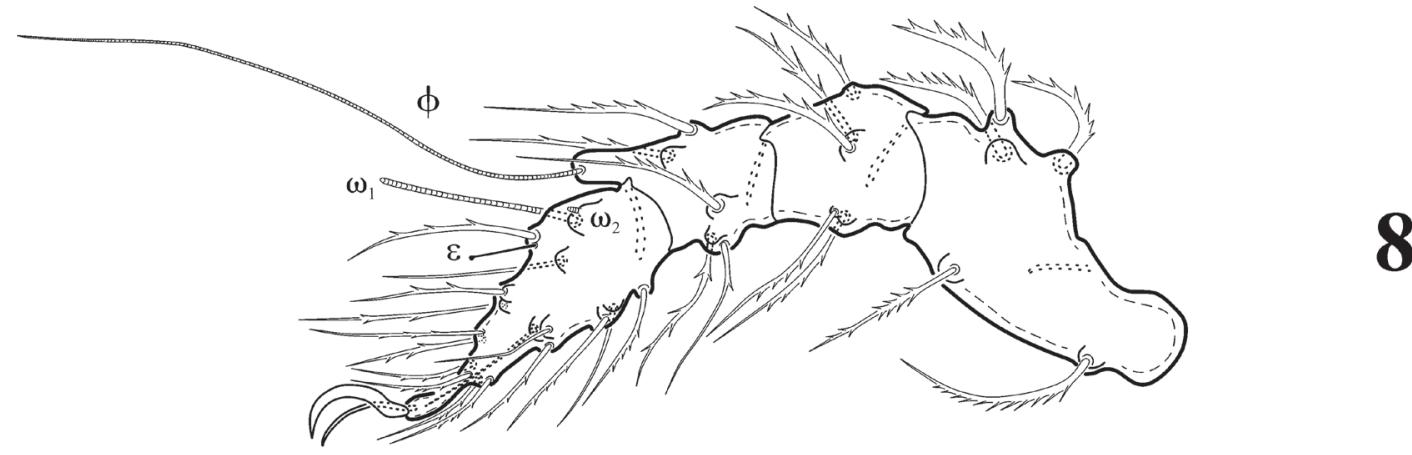

8
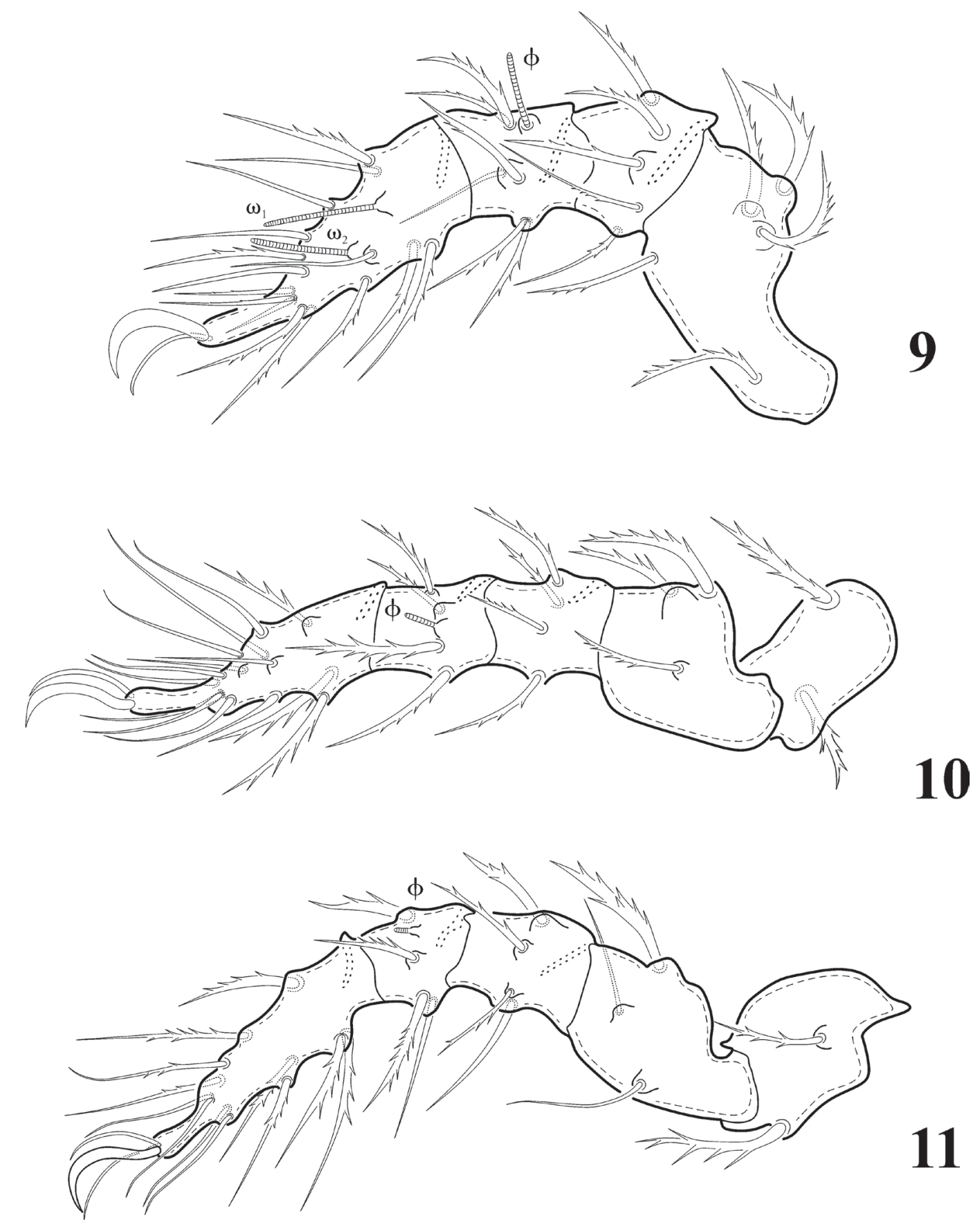

Figuras 8-11. Phyllozetes aldretei sp. nov. patas I-IV, vista antiaxial. Escala $30 \mu \mathrm{m}$. 